\title{
Effective Hamiltonian Approach to Catalytic Activity of Transition Metal Complexes
}

\author{
A. M. TOKMACHEV, A. L. TCHOUGRÉEFF, I. A. MISURKIN \\ Karpov Institute of Physical Chemistry, Vorontsovo pole 10, Moscow 103064, Russia
}

Received 9 October 2000; accepted 21 February 2001

\begin{abstract}
The application of an effective electron Hamiltonian approach to the description of the electronic structure of transition metal complexes with chemically active ligands is analyzed. This approach is implemented in a computational code. The evolution of the electronic structure along a path of isomerization of quadricyclane to norbornadiene in the coordination sphere of Co-tetraphenylporphyrin is considered. In addition, the electronic states of atomic oxygen coordinated to transition metal oxides and metal porphyrins are studied. (c) 2001 John Wiley \& Sons, Inc. Int J Quantum Chem 84: 99-109, 2001
\end{abstract}

Key words: effective Hamiltonian; catalysis; oxygen adsorption; transition metal oxides; metal porphyrines

\section{Introduction}

$\mathbf{T}$ he transition metal compounds (TMCs) are widely used as catalysts in many important practical processes such as oxygenation, $\mathrm{CO}$ insertion, and isomerization of hydrocarbons [1]. The overall performance of catalysis can be limited by different stages of the process like transport of reagents and products or by the chemical transformation itself to be completed in the coordination sphere of the transition metal atom. Even when diffusion is the limiting stage in the overall process, ac.ru.

Correspondence to: A. L. Tchougréeff; e-mail: andrei@cc.nifhi.

Contract grant sponsor: INTAS.

Contract grant number: 94-4089. a catalyst must interact with reactants in a way that ensures the high rate of the chemical stage of transformation of reactants to products, which is a limiting step in the absense of catalyst. For that reason the key problem in quantum chemical understanding of the TMC catalysis is disclosing the factors controlling the form of the potential energy surface (PES) of the reactants/products complex with a catalyst (catalytic complex) compared to that of free reactants.

The construction of the PES is a complicated problem and the complications have a 2 -fold origin. First, the TMC-based catalysts (CTMCs) are usually rather large systems: this is true both for solid-state heterogeneous catalysts, for enzymes, and for their biomimetic models. Second, an essential feature of the electronic structure of the TMCs (including CTMCs which comprise catalyst and re- 
actants/products molecules attached to the latter) is the d shell which is usually believed to serve as an electron donor or acceptor for the reactants [2]. Also, interactions between reactants and catalyst can change not only charge but also the spin states of both, getting around the spin conservation rules restricting the reaction [3]. High correlation of electrons in the $\mathrm{d}$ shell of the transition metal atom in complexes leads to problems for self-consistent field (SCF)-based methods when the latter are applied to spin and symmetry properties of the ground state of the TMCs as well as to d-d electron spectra. The situation in CTMCs is even more complicated since with these not only d electrons but also electrons in the cleaving or forming bonds in the reactants/products ligands must be treated by taking account of correlations. The density functional theory-based methods have problems analogous to those of other one-electron approaches. For example, they predict the wrong ground state of nickel oxide. Here the problem is not purely numerical, but one of reproducing the qualitative features of the electronic structure of the TMC. In addition, strongly correlated calculations of the whole catalytic system are not available even for one point of the configuration space; the construction of the PESs is really an incredible task. At the same time the correlated description is necessary only for a small part of the whole system, i.e., for the reaction center. The situation is typical for applying hybrid methods when different parts of the system are described with different levels of approximation (usually the combination of quantum and molecular mechanical approaches is used).

It is important to address not the purely computational problem of describing TMC catalysis but also the problem of "understanding" the latter. The analogous reasons moved Ruedenberg [4] to formulate a dichotomy between the high-precision numerical calculation and clearness of the qualitative description. Indeed, to reach quantitative accuracy we use model Hamiltonians as exact as possible and try to solve the corresponding eigenvalue/eigenfunction problem as precisely as possible. This way sometimes leads us to numerical exactness but very rarely to qualitative understanding. On the other hand, understanding requires the use of totally different tools, i.e., the phenomenological Hamiltonians and approximate wave functions related to them. The most renowned example of a phenomenological Hamiltonian is, of course, the Heisenberg Hamiltonian, which contains a single parameter $J$, and almost no electrons-they are re- placed by spins. An example somewhat closer to our topic is the phenomenological Hamiltonian of crystal field theory [5], which ignores all electrons except those in the $\mathrm{d}$ shell and replaces all possible effects of the environment by several parameters whose exact number depends on the symmetry of the complex. Everyone knows that these phenomenological approaches may be easily questioned: why the number of parameters is that; why their numerical values are those, and so on. But nevertheless, they do give insight into the nature of phenomena.

It seems that this dichotomy cannot be overcome except with the use of a method which in fact resolves the contradiction between the model (even ab initio) and the phenomenological approach. That is the effective Hamiltonian method. It is supposed to bridge the gap between a model Hamiltonian which is assumed to be exact (it may be the ab initio one or not, but for the purpose at hand it is considered as the exact one) and a phenomenological Hamiltonian reflecting the principal qualitative features of the process or object under consideration. The main features of the effective Hamiltonian approach as applied to rationalization of the phenomenological approaches are (1) a reduced number of electron variables, (2) principal calculability of the parameters, and (3) a form coinciding with that of the phenomenological Hamiltonian.

This general scheme has been recently implemented in the effective crystal field (ECF) method [6], which represents the hybrid approach for describing the electronic spectra of $\mathrm{d}-\mathrm{d}$ excitations in the TMCs. It exploits the group function formalism and the Löwdin partitioning method to separate the electronic variables relevant to the $\mathrm{d}$ shell and the ligands, respectively. It allows one to take into account both the electron correlations in the $\mathrm{d}$ shell and the Coulomb and resonance interactions between the $\mathrm{d}$ shell and the ligands. Calculations of a wide range of electronic spectra of TMCs have shown that the ECF method allows the description of the $\mathrm{d}-\mathrm{d}$ transitions in satisfactory agreement (within $1000 \mathrm{~cm}^{-1}$ ) with experimental results [7-9]. At the same time there are essential restrictions imposed on the problems which can be solved by the ECF method. They arise from two features of the ECF approach: the electron correlations in ligands are totally neglected and the number of electrons in the $d$ subsystem must be a good quantum number (i.e., approximately integers that coincide with the number of $d$ electrons in the corresponding free metal ion) to use the perturba- 
tion expansion in the effective Hamiltonian method correctly. Obviously, such restrictions do not allow use of the ECF method for description of catalytic processes (or, more commonly, for processes accompanied by significant modifications in electronic structure of ligands). The purpose of this work is to parallel the derivation that led to the ECF method to bridge the gap between the model and phenomenological description of catalysis by transition metal complexes and to bypass the difficulties cited above. To do so we first describe a phenomenological Hamiltonian relevant for description of catalysis by TMCs [10-13]. According to Refs. [10-13] the form of the phenomenological Hamiltonian is the sum of the Hamiltonians for the components, i.e., the reactants and catalyst and their interaction:

$$
\mathbf{H}=\mathbf{H}_{\text {react }}+\mathbf{H}_{\text {cat }}+\mathbf{H}_{\text {int }} .
$$

The phenomenological Hamiltonian for the reactants must describe several electronic states necessary to reproduce the transformation of the reactants to the products. Actually the Hamiltonian for reactants and that for the products is the same Hamiltonian but for different values of nuclear coordinates.

If the interaction between the catalyst and reactants/products vanishes, the eigenfunction of the total Hamiltonian becomes $\Phi_{\text {react }}^{i} \wedge \Phi_{\text {cat }}^{k}$ and the eigenvalues are simply sums of the eigenvalues of the subsystem Hamiltonians:

$$
\begin{aligned}
E^{i k} & =E_{\text {react }}^{i}+E_{\text {cat }}^{k} \\
E_{\text {react }}^{i} & =\left\langle\Phi_{\text {react }}^{i}\left|\mathbf{H}_{\text {react }}\right| \Phi_{\text {react }}^{i}\right\rangle, \\
E_{\text {cat }}^{k} & =\left\langle\Phi_{\text {cat }}^{k}\left|\mathbf{H}_{\text {cat }}\right| \Phi_{\text {cat }}^{k}\right\rangle .
\end{aligned}
$$

They have the same form as those for the free reactants/products but shifted by the corresponding catalyst energies. When the interaction is turned on the ground state of the catalytic complex, it becomes a linear combination which is sometimes called an "entangled" state [14]:

$$
\Psi^{0}=\sum_{i, k} A_{i k}^{0} \Phi_{\text {react }}^{i} \wedge \Phi_{\text {cat }}^{k}, \quad \sum_{i, k}\left(A_{i k}^{0}\right)^{2}=1 .
$$

When the interaction is turned on, the electronic energy becomes

$$
\begin{aligned}
E=\langle & \left.\Psi^{0}|\mathbf{H}| \Psi^{0}\right\rangle=\sum_{i, k}\left(A_{i k}^{0}\right)^{2}\left(E_{\text {react }}^{i}+E_{\text {cat }}^{k}\right) \\
+ & \sum_{i, k} \sum_{i^{\prime}, k^{\prime}} A_{i k}^{0} A_{i^{\prime} k^{\prime}}^{0} \\
& \times\left\langle\Phi_{\text {react }}^{i} \wedge \Phi_{\text {cat }}^{k}\left|\mathbf{H}_{\text {int }}\right| \Phi_{\text {react }}^{i^{\prime}} \wedge \Phi_{\text {cat }}^{k^{\prime}}\right\rangle,
\end{aligned}
$$

where the amplitudes of different basis product states can be estimated. The perturbative estimate for the amplitudes has the form

$$
A_{i k}^{0} \propto \frac{\left\langle\Phi_{\text {react }}^{i} \wedge \Phi_{\text {cat }}^{k}\left|\mathbf{H}_{\text {int }}\right| \Phi_{\text {react }}^{0} \wedge \Phi_{\text {cat }}^{0}\right\rangle}{\left(\left(E_{\text {react }}^{i}-E_{\text {react }}^{0}\right)+\left(E_{\text {cat }}^{k}-E_{\text {cat }}^{0}\right)\right)},
$$

so that the phenomenological Hamiltonian described above does its work: it gives a phenomenological description of transformation of reactants coordinated by a catalyst. The estimate for the amplitudes in Eq. (5) allows us to establish correlations between the physical properties of the free catalyst and its activity, which from our point of view justifies the phenomenological approach to catalysis proposed in Refs. [10-13]. The following sections focus on construction of a hybrid approach, taking into account equally the electron correlations in the $\mathrm{d}$-shell and some part of the ligands.

\section{Method}

The general way to derive an effective Hamiltonian for the partitioning interacting subsystems of a complex system is to use a combination of the McWeeny group function technique [15] and the Löwdin partition technique [16]. Let $\mathbf{H}$ be the Hamiltonian of the system consisting of two parts $A$ and $B$. Therefore, $\mathbf{H}$ can be represented as a sum of the Hamiltonians for bare $A$ and $B$ subsystems and the operator of interaction between subsystems:

$$
\mathbf{H}=\mathbf{H}_{A}+\mathbf{H}_{B}+V_{A B} .
$$

The exact wave function $\Psi$ is replaced by the antisymmetrized product of the wave functions of its components $A$ and $B: \Phi_{A} \wedge \Phi_{B}$. The entanglement of two subsystems is lost and to take it into account the original $\mathrm{ab}$ initio Hamiltonian $\mathbf{H}$ is replaced by the effective one $\left(\mathbf{H}^{\text {eff }}\right)$ acting in the space of the product functions $\Phi_{A} \wedge \Phi_{B}$. The requirement imposed on $\mathbf{H}^{\text {eff }}$ is that its eigenvalues coincide with those of the exact Hamiltonian $\mathbf{H}$. Denoting the projection operator on the space of product functions $\Phi_{A} \wedge \Phi_{B}$ as $P$ and the complementary projection operator as $Q$ $(=1-P)$, one obtains that

$$
\mathbf{H}^{\mathrm{eff}}=P \mathbf{H} P+P V_{A B} Q R(E) Q V_{A B} P,
$$

where the resolvent operator is

$$
R(E)=(E Q-Q \mathbf{H} Q)^{-1} .
$$

As is clear from analysis of the desirable features of the phenomenological Hamiltonian given in the Introduction the electron correlations must 
be covered in both the $\mathrm{d}$ shell and to some extent in the ligands. The hybrid scheme assumes division of the whole system into parts. The subsystem, which requires a highly correlated quantum chemical method, consists of the $d$ shell of the transition metal atom and of some electronic states of the ligands. The ligand variables can be chosen in a unified way.

Dividing a TMC in two subsystems, the d shell and the ligands (the $s$ and $p$ orbitals of the transition metal atom are ascribed to the ligands), is natural when the electronic structures of the ligands is intact. This separation is implemented in the ECF method, where the Hamiltonian is presented by a sum of the bare Hamiltonians of the subsystems and operators of Coulomb and resonance interaction between them:

$$
\mathbf{H}=\mathbf{H}_{d}+\mathbf{H}_{l}+V_{c}+V_{R} .
$$

The effective Hamiltonian for the $l$ subsystem can be approximated as a sum of the bare Hamiltonian for the $l$ subsystem and the interaction of the ligands with the Coulomb field induced by the d shell [6],

$$
\mathbf{H}_{l}^{\text {eff }}=\mathbf{H}_{l}+t r_{d}\left(P_{d} V_{c}\right),
$$

where $t r_{d}$ denotes the summation over the $\mathrm{d}$ shell variables and $P_{d}$ is the one-electron density matrix for the $d$ subsystem. The transition from the bare Hamiltonian for the ligands to the effective one [Eq. (10)] leads to renormalization of transition metal atom one-electron parameters:

$$
U_{i i}^{\text {eff }}=U_{i i}+\frac{n_{d}}{5} \sum_{\mu \in d} g_{\mu i},
$$

where $n_{d}$ is a number of electrons in the d shell of the bare transition metal ion. The core charge of the transition metal atom is also renormalized:

$$
Z_{M}^{\text {eff }}=Z_{M}-n_{d} .
$$

To describe the electronic structure of the ligand subsystem the SCF approach was used within the ECF formalism.

A common way to single out the one-electron states of the ligands responsible for chemical transformation is to select several highest occupied molecular orbitals (HOMOs) and lowest unoccupied molecular orbitals (LUMOs) of the reactants. These MOs are regarded as the $r$ (reactive) subsystem. Examples of such MOs are given in the next section. Procedures to select these MOs automatically or in a special way are implemented as well.

The selection of the $r$ subsystem leads to dividing the model ( $\mathrm{ab}$ initio) electronic Hamiltonian $\mathbf{H}$, which differs from that given by Eq. (9). The d shell and $r$ subsystem are now to be combined in the $(d \oplus r)$ subsystem for which electron correlations must be taken into account as completely as possible. The rest of the TMC [ $(l \ominus r)$ subsystem] is described by a low-level (SCF-based) method. Therefore, for these subsystems

$$
\mathbf{H}=\mathbf{H}_{d \oplus r}+\mathbf{H}_{l \ominus r}+V_{c}^{\prime}+V_{R^{\prime}}^{\prime}
$$

where $V_{c}^{\prime}$ and $V_{R}^{\prime}$ are the operators of Coulomb and resonance interactions between the subsystems $(d \oplus r)$ and $(l \ominus r)$. One may think that the operator $V_{c}^{\prime}$ is that part of the intersubsystem interaction which conserves the numbers of electrons in the respective subsystems, whereas $V_{R}^{\prime}$ by contrast transfers electrons from one subsystem to another. Two-electron transfers which in principle appear in the Hamiltonian are not very large and we omit them here for the sake of simplicity. The effective Hamiltonian for the $(d \oplus r)$ subsystem acting in the space of functions with fixed numbers of electrons in both subsystems is obtained by averaging the effective Hamiltonian [Eq. (7)] over the wave function of the $(l \ominus r)$ subsystem,

$$
\mathbf{H}_{d \oplus r}^{\mathrm{eff}}=\mathbf{H}_{d \oplus r}+\left\langle\left\langle V_{c}^{\prime}+V_{R R}^{\prime}\right\rangle\right\rangle_{l \ominus r},
$$

where we introduce the operator coupling two oneelectron transfers between the subsystems:

$$
V_{R R}^{\prime}=P V_{R}^{\prime} Q\left(E Q-Q H_{0} Q\right)^{-1} Q V_{R}^{\prime} P .
$$

The operator $P$ projects onto the subspace with a fixed number of electrons in the $(d \oplus r)$ and $(l \ominus r)$ subsystems. The operator $\mathbf{H}_{0}$ is the part of the Hamiltonian which is diagonal with respect to the operators $P$ and $Q$ (or more precisely with respect to the operator of the number of electrons in the subsystems):

$$
\mathbf{H}_{0}=\mathbf{H}_{d \oplus r}+\mathbf{H}_{l \ominus r}+V_{c}^{\prime} .
$$

The resolvent contribution [Eq. (15)] depends on energy. This dependence is, however, weak and will be omitted in our following considerations. It corresponds to the second order of the operator form of the Rayleigh-Schrödinger perturbation theory.

The effective Hamiltonian [Eq. (14)] depends on the wave function of the $(l \ominus r)$ subsystem. We use as an approximation that this subsystem is constructed from the MOs of the $l$ subsystem obtained by the SCF procedure with the effective Hamiltonian [Eq. (10)] excluding those ascribed to the $r$ subsystem. The effective Hamiltonian for the $(d \oplus r)$ subsystem can be written as a sum of one-electron 
and two-electron contributions [17]:

$$
\mathbf{H}_{d \oplus r}^{\mathrm{eff}}=\mathbf{H}_{d \oplus r}^{(1)}+\mathbf{H}_{d \oplus r}^{(2)} .
$$

Each of these contributions can be further subdivided. The one-electron contribution is

$$
\begin{aligned}
\mathbf{H}_{d \oplus r}^{(1)}=\sum_{\mu \in d} \mathbf{H}_{d \oplus r}^{(1) \mathrm{at}, \mu \mu} & +\sum_{\mu, v \in d} \mathbf{H}_{d \oplus r}^{(1) \mathrm{ECF}, \mu v}+\sum_{\substack{\mu \in d \\
i \in r}} \mathbf{H}_{d \oplus r}^{(1) \mathrm{res}, \mu i} \\
& +\sum_{i \in r} \mathbf{H}_{d \oplus r}^{(1) \mathrm{int}, i i}+\sum_{i, j \in r} \mathbf{H}_{d \oplus r}^{(1) \mathrm{dint}, i j}
\end{aligned}
$$

The Coulomb interaction of electrons occupying $\mathrm{d}$ orbitals with core and electron density populating in the $\mathrm{s}$ and $\mathrm{p}$ orbitals of a transition metal atom is

$$
\begin{aligned}
\mathbf{H}_{d \oplus r}^{(1) \mathrm{at}, \mu \mu}=\sum_{\sigma} d_{\mu \sigma}^{+} d_{\mu \sigma}\left(U_{d d}+P_{s s} g_{s d}\right. & \\
& \left.+\bar{g}_{p d} \sum_{\alpha \in p} P_{\alpha \alpha}\right),
\end{aligned}
$$

where $P_{a a}$ is the diagonal element of the density matrix of the $l$ subsystem [only for s and p orbitals of the transition metal atom in Eq. (19)]. The next contribution to Eq. (18) coincides with that of the ECF induced by the ligands [6]:

$$
\begin{aligned}
\mathbf{H}_{d \oplus r}^{(1) \mathrm{ECF}, \mu \nu}= & \sum_{\sigma} d_{\mu \sigma}^{+} d_{v \sigma}\left[\sum_{L}\left(P_{L}-Z_{L}\right) V_{\mu \nu}^{L}\right. \\
& \left.-\sum_{j \in l \ominus r} \beta_{\mu j} \beta_{\nu j}\left\{\frac{\left(1-n_{j}\right)^{2}}{\Delta E_{d j}}-\frac{n_{j}^{2}}{\Delta E_{j d}}\right\}\right] .
\end{aligned}
$$

The expression in square brackets corresponds to the Coulomb interaction of the $\mathrm{d}$ shell with the ligands averaged over the wave function of the $(l \ominus r)$ subsystem. Here $V_{\mu \nu}^{L}$ is the matrix element of this interaction and $P_{L}$ is the electron density on the ligand atom $L$ due to electrons of the $(l \ominus r)$ subsystem. The next contribution to Eq. (20) arises from resolvent contribution [Eq. (15)]. $\beta_{\mu j}$ is the corresponding resonance integral, $n_{j}$ is the occupancy of the $j$ th $\mathrm{MO}$ (equal to 1 if the $\mathrm{MO}$ is doubly occupied and 0 otherwise), and $\Delta E_{d j}$ and $\Delta E_{j d}$ are the energies of the one-electron transfer from the $\mathrm{d}$ shell to the $j$ th $\mathrm{MO}$ and vice versa.

The next contribution describes one-electron transfers between the $\mathrm{d}$ shell and the orbitals active in the chemical transformation. It changes the number of electrons in the $\mathrm{d}$ shell and in the $r$ subsystem:

$$
\mathbf{H}_{d \oplus r}^{(1) \mathrm{res}, \mu i}=\beta_{\mu i} \sum_{\sigma}\left(d_{\mu \sigma}^{+} r_{i \sigma}+r_{i \sigma}^{+} d_{\mu \sigma}\right) .
$$

The intra- $r$ subsystem one-electron energy is

$$
\mathbf{H}_{d \oplus r}^{(1) \text { int }, i i}=\left(\epsilon_{i}-\sum_{j \in r} n_{j}\left(2 J_{i j}-K_{i j}\right)\right) \sum_{\sigma} r_{i \sigma}^{+} r_{i \sigma},
$$

where $\epsilon_{i}$ is the energy of the $i$ th orbital in the $r$ subsystem and $J_{i j}$ and $K_{i j}$ are the Coulomb and exchange integrals between the $i$ th and $j$ th MOs. The last contribution to the one-electron part of the effective Hamiltonian is

$$
\mathbf{H}_{d \oplus r}^{(1) \operatorname{dint}, i j}=-n_{d} G_{d i j} \sum_{\sigma} r_{i \sigma}^{+} r_{j \sigma},
$$

where $G_{d i j}$ is the matrix element $(d d \mid i j)$.

Two-electron contributions can be also further subdivided:

$$
\begin{array}{r}
\mathbf{H}_{d \oplus r}^{(2)}=\sum_{\mu, v, \rho, \eta \in d} \mathbf{H}_{d \oplus r}^{(2) \mu v \rho \eta}+\sum_{\mu \in d, i, j \in r} \mathbf{H}_{d \oplus r}^{(2) \mu \mu i j} \\
+\sum_{i, j, k, l \in r} \mathbf{H}_{d \oplus r}^{(2) i j k l},
\end{array}
$$

where

$$
\begin{aligned}
\mathbf{H}_{d \oplus r}^{(2) \mu \nu \rho \eta} & =\frac{1}{2}(\mu \nu \mid \rho \eta) \sum_{\sigma \tau} d_{\mu \sigma}^{+} d_{\rho \tau}^{+} d_{\eta \tau} d_{\nu \sigma}, \\
\mathbf{H}_{d \oplus r}^{(2) \mu \mu i j} & =\frac{1}{2} G_{\mu i j} \sum_{\sigma \tau} d_{\mu \sigma}^{+} d_{\mu \sigma} r_{i \tau}^{+} r_{j \tau}, \\
\mathbf{H}_{d \oplus r}^{(2) i j k l} & =\frac{1}{2}(i j \mid k l) \sum_{\sigma \tau} r_{i \sigma}^{+} r_{k \tau}^{+} r_{l \tau} r_{j \sigma} ;
\end{aligned}
$$

we use a standard notation for the Coulomb matrix elements.

The number of one-electron states included in the $(d \oplus r)$ subsystem is relatively small (typically, less than 10). This space size allows use of the full configuration interaction method to solve the eigenproblem for the effective Hamiltonian [Eq. (14)]. We used unitary group analysis [18-20] to obtain the weights of the spin-adapted configurations in the ground and excited states of TMCs.

The energy of the combined system can be readily determined using the wave functions of the subsystems. Let us denote by $\Psi_{l \ominus r}$ the single determinant wave function of the $(l \ominus r)$ subsystem and by $\Psi_{d \oplus r}^{k}$ the $k$ th eigenfunction of the effective Hamiltonian [Eq. (14)]. Then, the electronic energy of the $k$ th state of the combined system is approximated by

$$
E^{k}=\left\langle\Psi_{d \oplus r}^{k}\left|\mathbf{H}_{d \oplus r}^{\text {eff }}\right| \Psi_{d \oplus r}^{k}\right\rangle+\left\langle\Psi_{l \ominus r}\left|\mathbf{H}_{l \ominus r}\right| \Psi_{l \ominus r}\right\rangle,
$$

i.e., the entanglement between subsystems leads to the appearance of the effective operator average in Eq. (26).

\section{Results and Discussion}

The method described in the previous section is implemented in the program suite CATALYST. 
It is mainly directed to analysis of the electronic structure of catalytic TMCs. In the present work we describe some initial applications of the above formalism to the description of the evolution of electronic structure in a series of processes: the isomerization of quadricyclane to norbornadiene and adsorption of atomic oxygen on the surfaces of transition metal oxides and metal porphyrines.

The isomerization reaction of quadricyclane to norbornadiene is the re-arrangement of a fourmembered ring into two double bonds. Without a catalyst this reaction is prohibited by symmetry according to the Woodward-Hoffmann rules [21]. More precisely, the HOMO and LUMO (having the $b_{1}$ and $b_{2}$ symmetries, respectively) of quadricyclane and norbornadiene intersect in the course of transformation. This reaction was studied experimentally [22, 23]. It was observed that some metal porphyrins are the catalysts of the isomerization of quadricyclane to norbornadiene.

The experimental data for this reaction were interpreted using the Mango-Schachtschneider (MS) theory [24]. The MS theory predicts the catalytic activity of Mn-phthalocyanine (Mn-pc) and inactivity of Co-tetraphenylporphyrin (Co-tpp) in contradiction with experiments [25]. The MS theory states that the orbital symmetry can be preserved by transfer of an electron pair from the HOMO of the reagent to the unoccupied orbital of the TMC catalyst of the same symmetry and, vice versa, of an electron pair from the occupied orbital of the catalyst to the LUMO of the reagent. The $d_{x z}$ and $d_{y z}$ orbitals of the Co-tpp (the ground state is doublet ${ }^{2} A_{1 g}$ ) having the symmetry of intersecting MOs of the reactants are fully occupied, leading to inactivity of Co-tpp in this reaction according to MS theory (but not by experiments). At the same time in Mn-pc these orbitals can take part in the redistribution of electrons between catalyst and reagents but experiments reveal inactivity of $\mathrm{Mn}$-pc in the isomerization reaction of quadricyclane to norbornadiene.

In other works $[10,11]$ another approach to analysis of the catalytic activity of TMCs was proposed. The catalytic activity of Co-tpp can be explained by the entanglement of the complex ground state ${ }^{2} A_{1}$ with the direct products of the ${ }^{4} A_{2 g}$ or ${ }^{4} B_{2 g}$ quartet states of Co-tpp and the ${ }^{3} A_{2}$ triplet state of the system quadricyclane-norbornadiene. This contribution can be obtained from the excited states of both the $\mathrm{d}$ shell of the catalyst and of the reactant. An interaction of these terms is ultimately

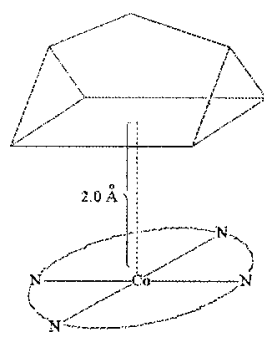

FIGURE 1. Scheme of quadricyclane adsorption on the Co-porphyrin.

caused by one-electron transfers between the subsystems [10, 11].

This model can be quantified by using the method described above. We studied the chemical reaction path from quadricyclane to norbornadiene in the coordination sphere of Co-tpp. The complex with quadricyclane is schematically drawn in Figure 1. The distance between the cobalt atom and the center of the four-membered ring was fixed at $2.0 \AA$. The intersecting MOs of the $b_{1}$ and $b_{2}$ symmetry (which are HOMO and LUMO for quadricyclane and norbornadiene) were included in the $r$ subsystem. The energies of excitation of the singlet ground state ${ }^{1} A_{1}$ to the triplet state ${ }^{3} A_{2}$ approximately equals $7 \mathrm{eV}$ both for quadricyclane and norbornadiene at their respective equilibrium geometries. Close to the transition state the ${ }^{1} A_{1}$ and ${ }^{3} A_{2}$ terms are almost degenerate. Our calculations have shown that the ground state of the catalytic complex is the doublet ${ }^{2} A_{1}$, which is mainly the direct product of the ground states of the catalyst and reactants. The overall weight of the states with charge transfer is about $1 \%$ along the whole reaction path. The doublet direct product of the low-lying quartet ${ }^{4} A_{2 g}$ of the Co-tpp and the ${ }^{3} A_{2}$ triplet of the quadricyclanenorbornadiene system contributes to the ground state. When the equilibrium geometric structures of quadricyclane and norbornadiene are under consideration the weight of this state is negligably small. At the same time, in the vicinity of reaction barrier this doublet became the major admixture to the leading ground state configuration. Its amplitude exceeds 0.15 . Therefore, the concept [10] loading the important role upon the excited states of the catalyst and reagents (and also the states with charge transfer) for the catalytic activity of the Co-tpp is in agreement with the numerical results obtained using the CATALYST package.

Oxygen is the most common oxidative agent. The catalytic reactions of oxidation are extensively used for detoxication of organic substances and of carbon 
oxide. The transition metal oxides or related materials are widely used oxidation catalysts. The mechanism of oxidation on these catalysts is, however, not well understood. At the same time it was shown that the activation energy of the process of the oxidation of dihydrogen is proportional to the oxygen binding energy to the catalyst surface [26]. On the basis of this dependence it was concluded [27] that the rate-determining step in the surface oxidation of $\mathrm{H}_{2}$ is

$$
\mathrm{H}_{2}+\mathrm{O}_{\text {surf }} \rightarrow \mathrm{H}_{2} \mathrm{O}_{\text {ad }} \rightarrow \mathrm{H}_{2} \mathrm{O} .
$$

The direct interaction (without catalyst) between free atomic oxygen and dihydrogen is prohibited because the spin state of oxygen is triplet and the spin state of dihydrogen is singlet. Therefore, one may expect that the process under consideration is going to be catalyzed if the state of the oxygen atom under interaction with a catalyst changes to singlet. The experimental studies of the state of the adsorbed oxygen atoms is difficult because it is also difficult to distinguish between the adsorbed oxygen and that from the bulk of the oxide. In the literature there exist only purely Coulomb estimations [28] and the indirect experimental data [29] for oxygen atoms on the $\mathrm{NiO}$ surface that predict the contribution of the $\mathrm{O}^{-}$form of the adsorbed oxygen to be significant. Moreover, electron spin resonance experiments manifest the formation of the $\mathrm{O}^{--}$anion-radicals during adsorption of dioxygen on oxide catalysts. It has been also concluded that the adsorbed anion-radical $\mathrm{O}^{--}$is highly reactive in the oxidation of carbon oxide and dihydrogen [30, 31].

The program package CATALYST allows elucidation of the state of oxygen atoms on the surface of transition metal oxides (Fig. 2). The important characteristic of the oxygen atom adsorbed is its spin state. The method gives the amplitudes of the configurations (characterized by their Young tableaus) in the wave function for the combined system (d shell and two orbitals of the oxygen atom). When both oxygen orbitals are singly occupied the

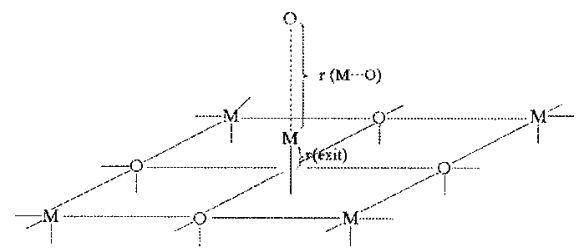

FIGURE 2. Scheme of oxygen adsorption on the surface of transition metal oxide. state of the oxygen in the configuration cannot be easily defined. Moreover, in this case one Young tableau does not allow us to determine the weights of singlet and triplet oxygen states. We must consider two corresponding Young tableaus and solve the system of two inhomogeneous linear equations. The required subduction coefficients are taken from Ref. [32]. The calculations were carried out using the cluster model of the solid catalyst. Transition metal oxide with the rock salt lattice type was modeled by a cluster containing 125 ions (62 ions of transition metal and 63 ions of oxygen). The $r$ subsystem was constructed from singly occupied $p_{x}$ and $p_{y}$ orbitals directed parallel to the surface. The charge on the adsorbed oxygen atom was determined as the sum of electrons transferred from the $\mathrm{d}$ shell to the $r$ subsystems taken with corresponding calculated weights for all configurations entering the ground state of the catalytic complex. The varied characteristics of the adsorption were the distance from the oxygen adsorbed to the transition metal ion and the distance from the transition metal ion to the surface. The dependencies of the ground state spin on these geometry parameters are plotted in the maps (Figs. 3-5) for cobalt, nickel, and iron oxides (dotted

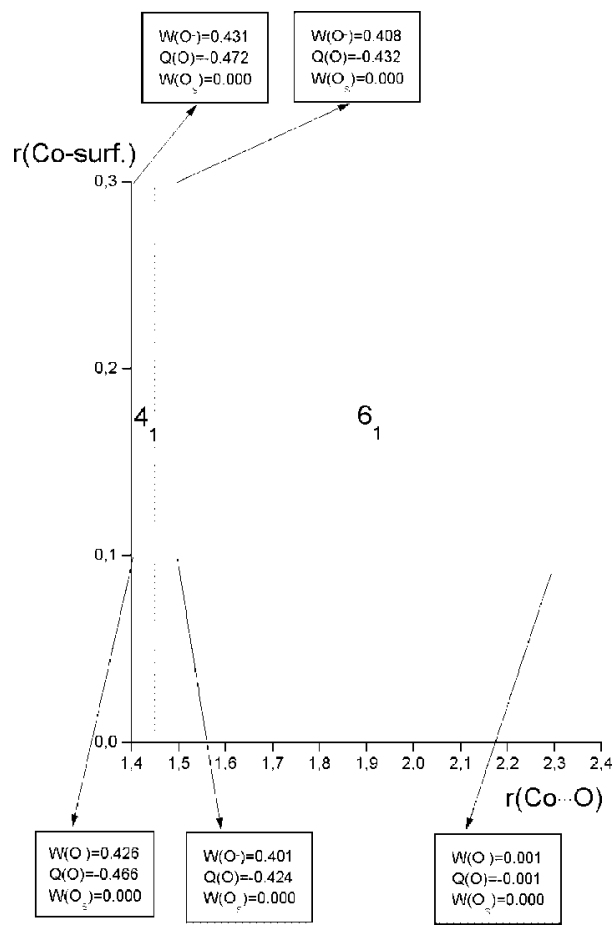

FIGURE 3. Spin states of the system $\mathrm{O} \ldots \mathrm{CoO}$, charge $[Q(O)]$, and weights of the states $\mathrm{O}^{-}$and $\mathrm{O}$ (singlet) $\left[\mathrm{W}\left(\mathrm{O}^{-}\right)\right.$and $\left.\mathrm{W}\left(\mathrm{O}_{\mathrm{s}}\right)\right]$ of the adsorbed oxygen atom as functions of distances $r(\mathrm{O} \ldots \mathrm{Co})$ and $r$ (Co-surface). 


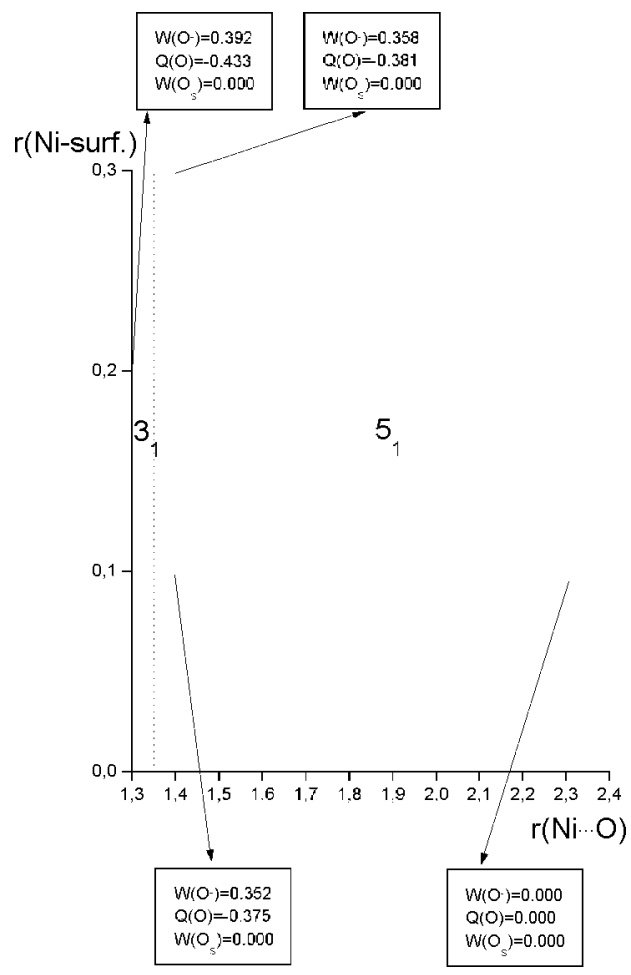

FIGURE 4. Spin states of the system $\mathrm{O} \ldots \mathrm{NiO}$, charge $[\mathrm{Q}(\mathrm{O})]$, and weights of the states $\mathrm{O}^{-}$and $\mathrm{O}$ (singlet) $\left[\mathrm{W}\left(\mathrm{O}^{-}\right)\right.$and $\left.\mathrm{W}\left(\mathrm{O}_{\mathrm{s}}\right)\right]$ of the adsorbed oxygen atom as functions of distances $r(\mathrm{O} \cdots \mathrm{Ni})$ and $r(\mathrm{Ni}$-surface).

lines denote approximate borders between different spin states).

For cobalt(II) oxide this map is relatively simple. The major part of the map (including large $\mathrm{Co}-\mathrm{O}_{\text {ads }}$ distances) corresponds to the overall sextet state. The main contribution to the ground state is the direct product of the quartet state of the $d$ shell (this contribution naturally corresponds to the ground state of the transition metal ion on the surface of oxide) and the triplet state of atomic oxygen. The weight of the ionic configurations with an electron transferred from the $\mathrm{d}$ shell to oxygen increases with decreasing the distance $\mathrm{Co}-\mathrm{O}_{\text {ads. }}$. The total charge on the oxygen atom is 0 for large $r\left(\mathrm{Co}-\mathrm{O}_{a d s}\right)$ and reaches -0.42 for $r\left(\mathrm{Co}-\mathrm{O}_{\mathrm{ads}}\right)=1.5 \AA$ and $r(\mathrm{Co}-$ surface $)=0.0 \AA$. The growth of the $r(\mathrm{Co}-$ surface $)$ to $0.3 \AA$ leads to an increase in the absolute charge on the oxygen atom of merely 0.01 . When $r(\mathrm{Co}-$ $\left.\mathrm{O}_{\mathrm{ads}}\right)=1.4 \AA$ the ground state of the catalytic complex switches to quartet with a leading contribution from the direct product of the doublet state of the Co ion on the oxide surface and triplet state of the oxygen atom. The charge on the oxygen atom in this state is -0.46 . The contribution from the con-

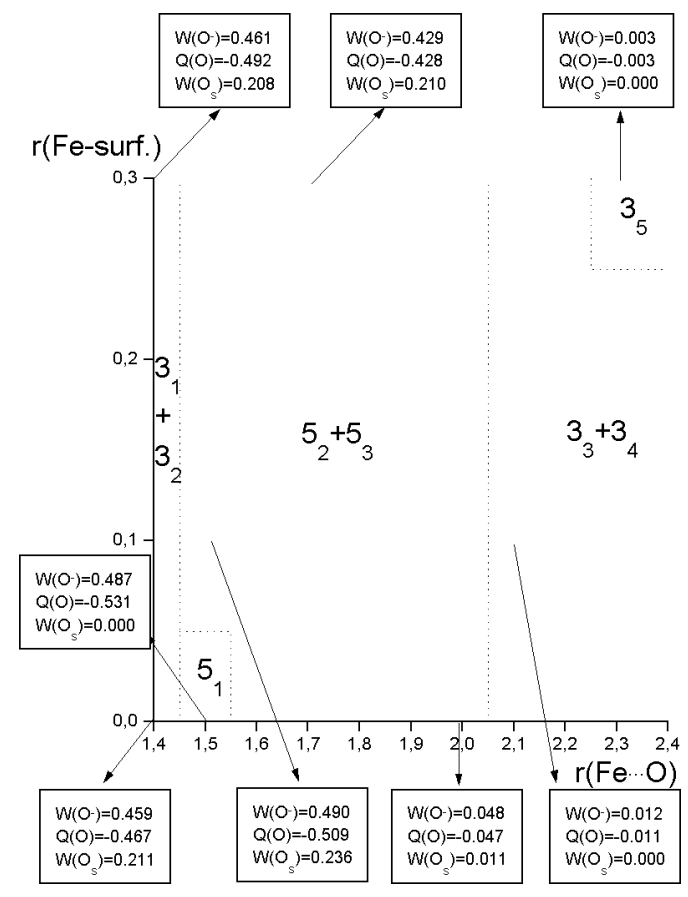

FIGURE 5. Spin states of the system $\mathrm{O} \ldots \mathrm{FeO}$, charge $[\mathrm{Q}(\mathrm{O})]$, and weights of the states $\mathrm{O}^{-}$and $\mathrm{O}$ (singlet) $\left[\mathrm{W}\left(\mathrm{O}^{-}\right)\right.$and $\left.\mathrm{W}\left(\mathrm{O}_{\mathrm{s}}\right)\right]$ of the adsorbed oxygen atom as functions of distances $r(\mathrm{O} \cdots \mathrm{Fe})$ and $r(\mathrm{Fe}-$ surface $)$.

figurations with a singlet oxygen is negligibly small. It can be stated that the value of $r$ (Co-surface) is not significant for defining the electronic state of adsorbed oxygen atoms on the surface of cobalt oxide.

For nickel(II) oxide the spin-state map is similar to that for cobalt(II) oxide. The quintet ground state is characteristic for the most part of the map. The main contribution to this state comes from the triplet configuration of oxide and the triplet configuration of atomic oxygen. For small distances $r\left(\mathrm{Ni}-\mathrm{O}_{\mathrm{ads}}\right)$ the ground state of the catalytic complex the triplet (the direct product of the singlet state of oxide and the triplet state of oxygen is the main contribution). It can be noticed that the absolute charge on the oxygen adsorbed on the nickel oxide $[-0.37$ for $\left.r\left(\mathrm{Ni}-\mathrm{O}_{\text {ads }}\right)=1.4 \AA\right]$ is smaller than that for the oxygen adsorbed on the cobalt oxide.

The spin map for the oxygen adsorption on the iron(II) oxide is much richer than those for cobalt(II) and nickel(II) oxides. In this case the exit of the metal atom from the surface plane affects the overall spin state and the state of oxygen in the expansion. If the distance $r\left(\mathrm{Fe}-\mathrm{O}_{\mathrm{ads}}\right) \geq 2.1 \AA$, then the ground state of the catalytic complex is triplet (obtained from the quintet state of $\mathrm{FeO}$ and the triplet state of atomic oxygen). For small distances $r(\mathrm{Fe}-$ 
surface) the ground state is degenerate $\left(3_{3}+3_{4}\right)$. When both $r\left(\mathrm{Fe}-\mathrm{O}_{\mathrm{ads}}\right)$ and $r(\mathrm{Fe}-$ surface $)$ are large enough, the ground state is nondegenerate $\left(3_{5}\right)$. The absolute charge on the oxygen atom for these triplet states does not exceed 0.01 . When $r\left(\mathrm{Fe}-\mathrm{O}_{\text {ads }}\right)$ ranges between 1.5 and $2.0 \AA$ the ground state of the catalytic complex is quintet. In the small region for the $r\left(\mathrm{Fe}-\mathrm{O}_{\mathrm{ads}}\right)$ distance close to $1.5 \AA$ and the $r(\mathrm{Fe}-$ surface) distance close to zero, the quintet ground state $\left(5_{1}\right)$ is nondegenerate and is formed mainly by the triplet states of the iron oxide and atomic oxygen. The charge on the oxygen atom in this state is rather large and equals -0.53 . Another quintet state $\left(5_{2}+5_{3}\right)$ is doubly degenerate. The decrease of the distance $r\left(\mathrm{Fe}-\mathrm{O}_{\mathrm{ads}}\right)$ from 2.0 to $1.5 \AA$ results in an increase in the absolute charge on the oxygen atom from 0.05 to 0.51 . Moreover, the contribution to the ground state from the states with singlet oxygen exceeds $10 \%$ for $r\left(\mathrm{Fe}-\mathrm{O}_{\mathrm{ads}}\right)=1.5 \AA$. If $r\left(\mathrm{Fe}-\mathrm{O}_{\mathrm{ads}}\right) \leq$ $1.4 \AA$ the ground state of the complex is a doubly degenerate triplet $\left(3_{1}+3_{2}\right)$. It is interesting that the absolute charge on the atomic oxygen in this state $\left[-0.47\right.$ for $\left.r\left(\mathrm{Fe}-\mathrm{O}_{\mathrm{ads}}\right)=1.4 \AA\right]$ is lower even than that for the quintet state with greater distance $r(\mathrm{Fe}-$ $\mathrm{O}_{\mathrm{ads}}$ ). At the same time the contribution from the configurations with singlet oxygen is greater than that for the quintet states.

The conclusion can be drawn that the state of noncharged oxygen adsorbed on the surface of cobalt, nickel, and iron oxides is predominantly triplet. The essential contribution of the singlet oxygen is observed only for iron(II) oxide for small distances $r\left(\mathrm{Fe}-\mathrm{O}_{\mathrm{ads}}\right)$. At the same time the configurations with negatively charged oxygen essentially contribute to the ground state of the catalytic complex. In the case of iron(II) oxide these configurations have the most weight in the ground state. It is experimentally well established that $\mathrm{CoO}$ and $\mathrm{NiO}$ are good catalysts of the oxidation by atomic oxygen. As is shown the singlet oxygen is not formed on their surfaces. Therefore, the important role in the catalytic process is given to the negatively charged states of the oxygen in accordance with the experiment-based conclusions [30,31].

The transition metal porphyrins can be also used as catalysts for oxidation. These species have been extensively studied both experimentally $[33,34]$ and theoretically [35-38]. It is interesting to compare the spin maps of the atomic oxygen coordinated to the transition metal atom of the metalloporphyrine and to the surface ion of an oxide. The formal difference is in the environment of the transition metal atom $[(l \ominus r)$ subsystem]. We compared cobalt, iron,

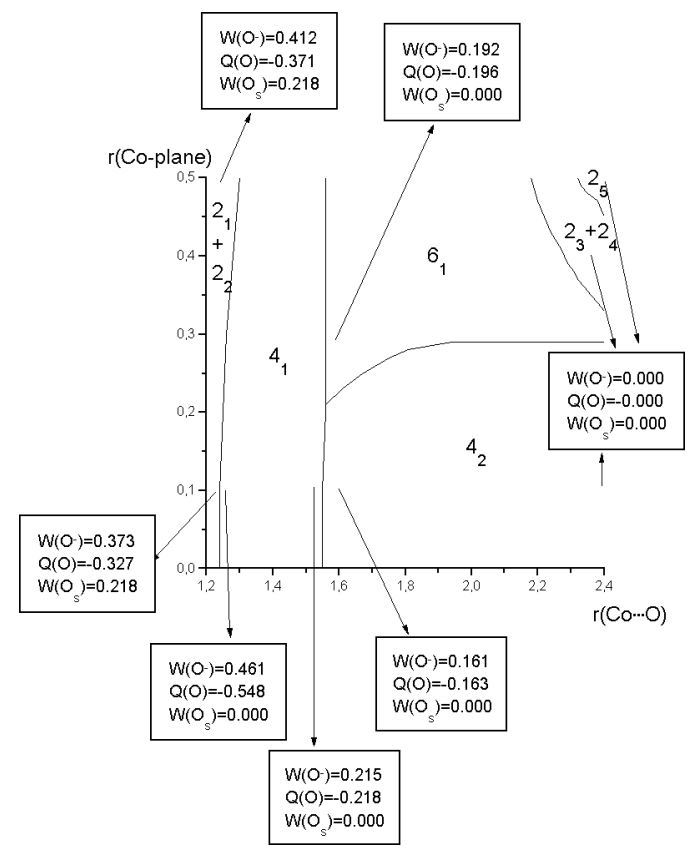

FIGURE 6. Spin states of the system O...CoP, charge $[Q(O)]$, and weights of the states $\mathrm{O}^{-}$and $\mathrm{O}$ (singlet) $\left[\mathrm{W}\left(\mathrm{O}^{-}\right)\right.$and $\left.\mathrm{W}\left(\mathrm{O}_{s}\right)\right]$ of the adsorbed oxygen atom as functions of distances $r(\mathrm{O} \ldots \mathrm{Co})$ and $r(\mathrm{Co}-$ plane $)$.

and manganese compounds. The spin maps for the atomic oxygen adsorbed on these metal porphyrins are shown in Figures 6-8. When we look at the state map for $\mathrm{CoP}=\mathrm{O}$ we see that it is mainly occupied with the states where the oxygen atom is largely in its triplet and the radical-anion states. Only at unrealistically short $\mathrm{Co}-\mathrm{O}$ distances do states with a significant contribution of singlet oxygen appear. What is particularly important is that the borders between the areas are sharp so that very small variations in the Co-O separation drastically change the state of the coordinated oxygen atom. This feature is characteristic for all maps. The map for $\mathrm{FeP}=\mathrm{O}$ is somewhat richer, and the area occupied by the states with significant contributions of singlet oxygen atoms is large. However there is no place where a noticeable contribution of the $\mathrm{O}^{2-}$ dianion can be found although it is frequently identified to explain things in the chemistry of monooxygenase enzymes and their biomimetic models [39].

The most interesting finding from that point of view is the map for $\mathrm{MnP}=\mathrm{O}$. It is rather simple with only one state seen. However everywhere we can see a noticeable contribution of the singlet oxygen atom. The pictures obtained for two ends of this short range $\mathrm{Mn}, \mathrm{Fe}$, and $\mathrm{Co}$, however, correspond well to the well-known observation that all 


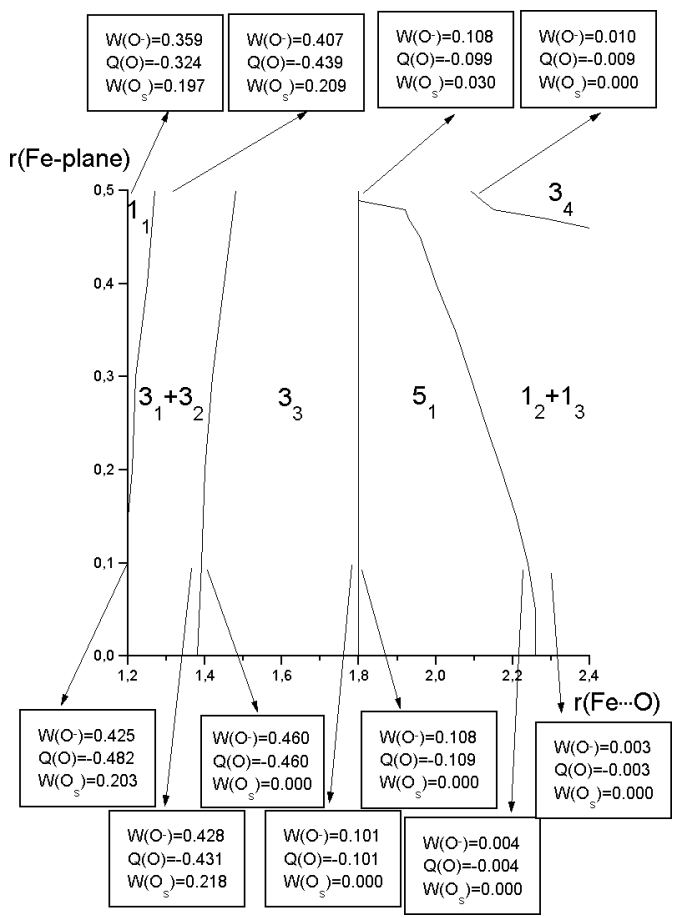

FIGURE 7. Spin states of the system $\mathrm{O} \ldots \mathrm{FeP}$, charge $[\mathrm{Q}(\mathrm{O})]$, and weights of the states $\mathrm{O}^{-}$and $\mathrm{O}$ (singlet) $\left[\mathrm{W}\left(\mathrm{O}^{-}\right)\right.$and $\left.\mathrm{W}\left(\mathrm{O}_{s}\right)\right]$ of the adsorbed oxygen atom as functions of distances $r(\mathrm{O} \ldots \mathrm{Fe})$ and $r(\mathrm{Fe}-$ plane $)$.

these metal porphyrines may serve as catalysts for a variety of oxygenation processes. However, $\mathrm{CoP}$ is known to direct the process toward a radical pattern (many oxygenation products, chain reaction etc.), but $\mathrm{MnP}$ directs the reaction to a single insertion product whether it goes about monoxygenation of olefines or alkanes. In this respect FeP occupies an intermediate position.

\section{Conclusions}

The reactions in the coordination sphere of transition metal atoms are in common use. The development of the quantum chemical methods calculating the electronic states and the energies of the catalytic complexes is very important. There are many problems in the construction of such approaches due to the necessity to take into account electron correlations in the $\mathrm{d}$ shell and in the reactants simultaneously. We constructed a method which uses different levels of approximation for the reactive part of system and the environment (ligands). The method is implemented with a program package.

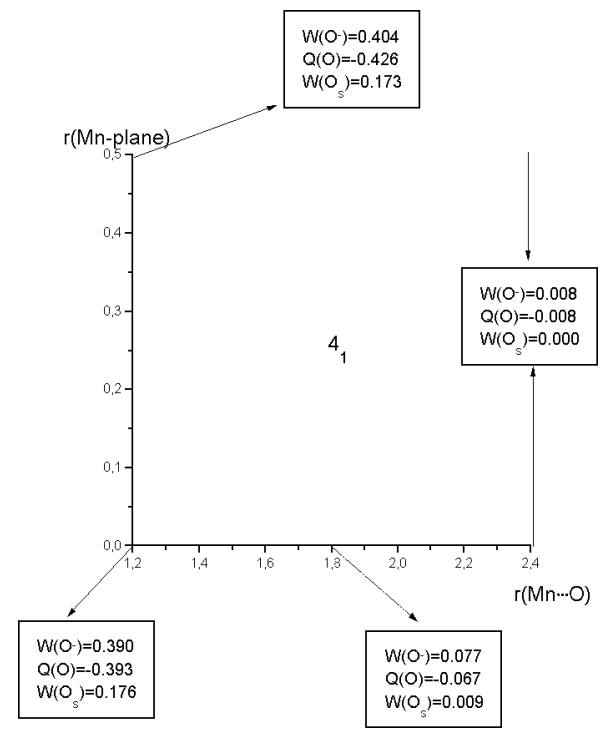

FIGURE 8. Spin states of the system $\mathrm{O} \cdots \mathrm{MnP}$, charge $[Q(O)]$, and weights of the states $\mathrm{O}^{-}$and $\mathrm{O}$ (singlet) $\left[\mathrm{W}\left(\mathrm{O}^{-}\right)\right.$and $\left.\mathrm{W}\left(\mathrm{O}_{s}\right)\right]$ of the adsorbed oxygen atom as functions of distances $r(\mathrm{O} \cdots \mathrm{Mn})$ and $r(\mathrm{Mn}$-plane).

This approach was applied to a series of experimental situations. The catalytic activity of Co-tpp in the isomerization reaction of quadricyclane to norbornadiene is explained by an admixture of the excited states of the quadricyclane-norbornadiene system and Co-tpp and affirmed by numerical calculations. The states of the oxygen adsorbed on the transition metal oxides are calculated and the experimental conclusion about important role of the states with electron transfer from the $d$ shell to the oxygen is confirmed.

\section{ACKNOWLEDGMENTS}

This work was supported by the INTAS through Grant 94-4089. It is a part of the Ph.D. thesis of A.M.T. One of us (A.M.T.) acknowledges financial support from the Haldor Topsøe A/S.

\section{References}

1. Masters, C. Homogeneous Transition-Metal Catalysis; Chapman and Hall: London, 1981.

2. Garner, W. E. In Advances in Catalysis and Related Subjects; Academic Press: New York, 1957; Vol. IX.

3. Nakamura, A.; Tsutsui, M. Principles and Applications of Homogeneous Catalysis; Wiley-Interscience: New York, 1980.

4. Ruedenberg, K. Rev Mod Phys 1962, 34. 
5. Jorgensen, C. K. Modern Aspects of Ligand Field Theory; North-Holland: Amsterdam, 1971.

6. Soudackov, A. V.; Tchougréeff, A. L.; Misurkin, I. A. Theor Chim Acta 1992, 83, 389.

7. Soudackov, A. V.; Tchougréeff, A. L.; Misurkin, I. A. Int J Quantum Chem 1996, 58, 161.

8. Soudackov, A. V.; Tchougréeff, A. L.; Misurkin, I. A. Zh Fiz Khim 1994, 68, 1256.

9. Tokmachev, A. M.; Tchougréeff, A. L. Chem Phys Rep 1999, $18,163$.

10. Tchougréeff, A. L.; Misurkin, I. A. Chem Phys 1989, 133, 77.

11. Tchougréeff, A. L.; Misurkin, I. A. Dokl Akad Nauk USSR 1986, 291, 1177.

12. Tchougréeff, A. L. Int J Quantum Chem 1996, 57, 413.

13. Tchougréeff, A. L. Int J Quantum Chem 1996, 58, 67.

14. Schumacher, B. Phys Rev A 1995, 51, 2738.

15. McWeeny, R. Methods of Molecular Quantum Mechanics, 2nd ed.; Academic Press: London, 1992.

16. Löwdin, P.-O. J Math Phys 1962, 3, 969.

17. Tokmachev, A. M.; Tchougréeff, A. L. Russ J Phys Chem 2000, 74, 58

18. Paldus, J. In Theoretical Chemistry, Advances and Perspectives; Eyring, H.; Henderson, D., Eds.; Academic Press: New York, 1976; Vol. 2.

19. Harter, W. G.; Patterson, C. W. Phys Rev A 1976, 13, 1067.

20. Harter, W. G.; Patterson, C. W. A Unitary Calculus for Electronic Orbitals; Springer-Verlag: Heidelberg, 1976; Vol. 49.

21. Woodward, R. B.; Hoffmann, R. J Am Chem Soc 1965, 87, 395.
22. Hogeveen, H.; Nusse, B. J. Tetrahedron Lett 1973, 3667.

23. Bishop, K. S. Chem Rev 1976, 76, 461.

24. Mango, F. D. Adv Catal 1969, 20, 291.

25. Manassen, J. J Catal 1970, 18, 38.

26. Boreskov, G. K. Kinet Katal 1967, 8, 1020.

27. Boreskov, G. K. In Catalysis-Science and Technology; Anderson, J. R.; Boudart, M., Eds.; Springer-Verlag: Berlin, Heidelberg, 1982; Vol. 3.

28. Bielanski, A.; Haber, J. Catal Rev 1979, 19, 1.

29. Bielanski, A.; Najbar, M. J Catal 1972, 25, 398.

30. Shwets, V. A.; Kazanskii, V. B. J Catal 1972, 25, 123.

31. Naccache, C. Chem Phys Lett 1971, 11, 323.

32. Patterson, C. W.; Harter, W. G. Phys Rev A 1977, 15, 2372.

33. Jameson, G. B.; Molinaro, F.; Ibers, J. A.; Collman, J. P.; Brauman, I. J.; Rose, E.; Suslick, K. S. J Am Chem Soc 1980, 102, 3224.

34. Steiger, B.; Baskin, J. S.; Anson, F. C.; Zewail, A. H. Angew Chem Int Ed 2000, 39, 257.

35. Dedieu, A.; Rohmer, M.-M.; Bernard, M.; Veillard, A. J Am Chem Soc 1976, 98, 3717.

36. Jewsbury, P.; Yamamoto, S.; Minato, T.; Saito, M.; Kitagawa, T. J Phys Chem 1995, 99, 12677.

37. Havlin, R. H.; Godbout, N.; Salzmann, R.; Wojdelski, M.; Arnold, W.; Schulz, C. E.; Oldfield, E. J Am Chem Soc 1998, 120, 3144

38. Rovira, C.; Parrinello, M. Int J Quantum Chem 2000, 80, 1172.

39. Meunier, B. Chem Rev 1992, 92, 1411. 